\section{LÚPUS ERITEMATOSO SISTÊMICO: ACOMETIMENTO CUTÂNEO/ARTICULAR}

Autoria: Sociedade Brasileira de Reumatologia

Participantes: Sato El, Bonfá ED, Costallat LTL, Silva NA, Brenol JCT, Santiago MB, Szajubok JCM, Rachid-Filho A, Barros RT, Vasconcelos $M$.

Método de coleta de evidências: Oito reumatologistas que trabalham em serviços que atendem grande número de pacientes com lúpus eritematoso sistêmico, alguns dos quais têm pesquisa e publicações científicas nesta área, foram convidados a participar do grupo de trabalho. Também foram convidados um nefrologista e uma dermatologista que atuam em grandes centros universitários, com grande experiência no atendimento destes pacientes. Todos se reuniram para discutir o tratamento das diferentes manifestações da doença, subdivididos em quatro grupos de trabalho, cada qual ficando responsável por buscar a melhor evidência para o tratamento de um ou mais comprometimentos da doença. A última edição de Dubois's lupus erythematosus, editado por Wallace D e Hahn B, em 200I, foi utilizada como base da discussão. Trabalhos publicados nos últimos cinco anos foram pesquisados no banco de dados do Medline. Devido à freqüência e à heterogeneidade de manifestações da doença, a maioria dos trabalhos terapêuticos não contempla grande casuística, nem é randômica e controlada. Como as manifestações e a gravidade da doença variam em diferentes grupos populacionais, devemos avaliar com cuidado os estudos realizados em grupos populacionais distintos.

\section{Graus de recomendação e força de evidência científica:}

A: Estudos experimentais e observacionais de melhor consistência.

B: Estudos experimentais e observacionais de menor consistência.

C: Relatos de casos (estudos não controlados).

D: Opinião desprovida de avaliação crítica, baseada em consensos, estudos fisiológicos ou modelos animais.

Conflito de interesse: Nenhum conflito de interesse declarado.

O lúpus eritematoso sistêmico é uma doença inflamatória crônica, multissistêmica, de causa desconhecida e de natureza auto-imune, caracterizada pela presença de diversos autoanticorpos. Evolui com manifestações clínicas polimórficas, com períodos de exacerbações e remissões. De etiologia não esclarecida, o desenvolvimento da doença está ligado à predisposição genética e aos fatores ambientais, como luz ultravioleta e alguns medicamentos. É uma doença rara, incidindo mais freqüentemente em mulheres jovens, ou seja, na fase reprodutiva, numa proporção de nove a dez mulheres para um homem, com prevalência variando de I 4 a 50/I 00.000 habitantes, em estudos norte-americanos'(D).

A doença pode ocorrer em todas as etnias e em todas as partes do mundo. Na prática, costuma-se estabelecer o diagnóstico utilizando-se os critérios de classificação propostos, em 1982, pelo American College of Rheumatology²(B), e revisados em 19973(D). O diagnóstico se baseia na presença de pelo menos 4 critérios dos I I citados a seguir:

।. Eritema malar: lesão eritematosa fixa em região malar, plana ou em relevo.

2. Lesão discóide: lesão eritematosa, infiltrada, com escamas queratóticas aderidas e tampões foliculares, que evolui com cicatriz atrófica e discromia.

3. Fotossensibilidade: exantema cutâneo como reação não usual à exposição à luz solar, de acordo com a história do paciente ou observado pelo médico.

4. Úlceras orais/nasais: úlceras orais ou nasofaríngeas, usualmente indolores, observadas pelo médico.

5. Artrite: artrite não erosiva envolvendo duas ou mais articulações periféricas, caracterizadas por dor e edema ou derrame articular.

6. Serosite: pleuris (caracterizada por história convincente de dor pleurítica, ou atrito auscultado pelo médico ou evidência de derrame pleural) ou pericardite (documentado por eletrocardiograma, atrito ou evidência de derrame pericárdico).

7. Comprometimento renal: proteinúria persistente $(>0,5 \mathrm{~g} /$ dia ou 3+) ou cilindrúria anormal.

8. Alterações neurológicas: convulsão (na ausência de outra causa) ou psicose (na ausência de outra causa).

9. Alterações hematológicas: anemia hemolítica ou leucopenia (menor que 4.000/ml em duas ou mais ocasiões) ou linfopenia (menor que 1.500/ml em duas ou mais ocasiões) ou plaquetopenia (menor que 100.000/ml na ausência de outra causa).

10. Alterações imunológicas: anticorpo anti-DNA nativo ou anti-Sm ou presença de anticorpo antifosfolípide baseado em: a) níveis anormais de $\lg G$ ou IgM anticardiolipina; b) teste positivo para anticoagulante lúpico ou teste falso positivo para sífilis, por no mínimo seis meses.

II. Anticorpos antinucleares: título anormal de anticorpo antinuclear por imunofluorescência indireta ou método equivalente, em qualquer época, e na ausência de drogas conhecidas por estarem associadas à síndrome do lúpus induzido por drogas.

Estes critérios foram desenvolvidos com o objetivo de uniformizar os estudos científicos da doença, e, embora raro, é possível termos pacientes que não apresentam quatro dos critérios de classificação.

A avaliação laboratorial reforça o diagnóstico quando se observar alterações tais como leucopenia, anemia, linfopenia, plaquetopenia e do sedimento urinário. De particular importância para o diagnóstico é a pesquisa de anticorpos ou fatores 
antinucleares por imunofluorescência indireta, utilizando como substrato as células HEp-2, conforme proposta do I Consenso Brasileiro Sobre Laudos de $\operatorname{FAN}^{4}(\mathbf{D})$. A positividade desse teste, embora não específico, serve como triagem devido a sua sensibilidade maior que 95\%, sendo altamente improvável a presença da doença se o teste resultar negativo ${ }^{2}(\mathbf{B})$. Nos raros casos da doença com pesquisa de FAN negativa, particularmente com lesões cutâneas fotossensíveis, recomenda-se a realização da pesquisa de anticorpos anti-SSa/ Ro. A pesquisa de anticorpos como anti-DNA nativo, anti-Sm, anti-RNP e células LE pode contribuir para melhor caracterização laboratorial do quadro. Por outro lado, a negatividade do FAN dispensaria a pesquisa desses auto-anticorpos.

\section{Medidas gerais}

Como parte importante da abordagem terapêutica, algumas medidas gerais são recomendadas, entre $\operatorname{elas}^{5}(\mathbf{D})$ :

I. Educação: informar, ao paciente e aos familiares, o que é a doença, sua evolução, riscos e os recursos disponíveis para diagnóstico e tratamento. Recomenda-se a necessidade de cumprimento das medidas estabelecidas pelo médico.

2. Apoio psicológico: transmitir otimismo e motivação para o tratamento, além de estimular os projetos de vida.

3. Atividade física: repouso nos períodos de atividade sistêmica da doença e medidas visando melhora do condicionamento físico (estimular atividade física regular).

4. Dieta: não há evidência científica de que os alimentos possam influenciar o desencadeamento ou evolução da doença. Recomenda-se a adoção de uma dieta balanceada, evitando-se excessos de sal, carboidratos e lipídios.

5. Proteção: contra luz solar e outras formas de irradiação ultravioleta.

6. Evitar: tabagismo.

\section{Tratamento medicamentoso}

O tratamento medicamentoso deve ser individualizado para cada paciente e dependerá dos órgãos ou sistemas acometidos, e da gravidade destes acometimentos. O tratamento de pacientes com comprometimento de múltiplos sistemas deverá ser orientado para o comprometimento mais grave. Quando houver manifestação que não responda a uma droga, pode ser necessário fazer uso concomitante de diversos medicamentos. Por exemplo, paciente com nefrite e lesões cutâneas pode necessitar de corticóide e imunossupressor para o tratamento da nefrite, associado ao uso de talidomida para o tratamento da lesão cutânea refratária.

Independentemente do órgão ou sistema afetado, o uso contínuo de antimaláricos como $4 \mathrm{mg} / \mathrm{kg} / \mathrm{dia}$ de difosfato de cloroquina ou $6 \mathrm{mg} / \mathrm{kg} / \mathrm{dia}$ de sulfato de hidroxicloroquina é indicado com a finalidade de reduzir atividade da doença e tentar poupar o uso de corticóides ${ }^{6}(\mathbf{B})$. A manutenção da droga em pacientes controlados reduz a possibilidade de novo surto de atividade ${ }^{7}(\mathbf{A})$. Melhora do perfil lipídico, como a redução do colesterol sérico $^{8}(\mathbf{B})$, a elevação do $H D L$-colesterol ${ }^{9}(\mathbf{B})$ e redução do risco de trombose ${ }^{10}(\mathbf{D})$ são benefícios adicionais atribuídos ao uso de antimaláricos.

Além dos antimaláricos, os glicocorticóides são as drogas mais utilizadas no tratamento. A dose de glicocorticóides varia de acordo com a gravidade de cada caso. Didaticamente as doses de glicocorticóides, tendo a prednisona como padrões, podem ser divididas em "'(D):

- Dose baixa: 0,125 mg/kg/dia;

- Dose moderada: 0, 125 a 0,5 mg/kg/dia;

- Dose alta: 0,6 a I mg/kg/dia;

- Dose muito alta: I a 2 mg/kg/dia;

- Pulsoterapia com glicocorticóides: injeção intravenosa de Ig de metilprednisolona ( $15-20$ mg/kg/dia) por três dias consecutivos.

Devido aos múltiplos efeitos colaterais, os glicocorticóides devem ser utilizados na dose efetiva para o controle da atividade da doença, e, assim que possível, deve haver redução gradual de sua dose. Embora haja grande variabilidade individual na sensibilidade aos glicocorticóides, está demonstrado que o uso de glicocorticóides de longa ação, como a dexametasona, é o mais deletério, devendo ser evitado de forma diária' ${ }^{2}(\mathbf{D})$. Nos pacientes que não conseguem atingir uma dose de manutenção de glicocorticóides aceitável, menor que $15 \mathrm{mg} / \mathrm{dia}$, está indicada a associação de outra droga para poupar glicocorticóides. Entre as drogas com comprovada ação poupadora de glicocorticóides, além dos antimaláricos ${ }^{6}(\mathbf{B})$ já mencionados, temos a azatioprina ${ }^{13}(\mathbf{D})$ e o metotrexate na manifestação cutânea e vasculítica ${ }^{14}(\mathbf{C})$. É importante o diagnóstico diferencial entre atividade da doença e infecção, lembrando da possibilidade de coexistência de ambas, assim como da presença de co-morbidades.

\section{Comprometimento cutâneo}

O tratamento vai depender da extensão do acometimento dermatológico e da gravidade das manifestações extracutâneas. Lembrar que nem toda lesão cutânea é LE específica, podendo decorrer de complicações do tratamento ou outras dermatoses concomitantes, requerendo condutas diagnósticas e terapêuticas diversas.

Fotoproteção: Considerando que a radiação ultravioleta $B$ é a principal causadora de fotossensibilidade e desencadeante das lesões cutâneas, protetores solares com FPS $=15$ devem ser utilizados em quantidade generosa pela manhã e reaplicados mais uma vez ao dia. $\bigcirc$ uso de bloqueadores solares de amplo espectro pode trazer benefício adicional pela capacidade de proteção contra UV-A, em algumas lesões cutâneas como as lesões subagudas ${ }^{15}(\mathbf{D})$, bem como no melhor prognóstico relacionado às lesões renal e trombocitopênica ${ }^{16}(\mathbf{B})$.

Nas lesões cutâneas localizadas, está indicada terapia tópica com corticóide não fluorado na face e áreas de flexão. Em lesões mais hipertróficas, indica-se corticóide fluorado, podendo ser aplicado sob a forma oclusiva ou de infiltração ${ }^{17}(\mathbf{D})$. 
As lesões cutâneas agudas geralmente respondem ao tratamento indicado para outras manifestações do LES, como corticosteróides e imunossupressores. Embora não haja estudos controlados, na prática clínica, os especialistas utilizam os antimaláricos para o tratamento do comprometimento cutâneo, quando não houver necessidade dessas drogas para outros comprometimentos. As lesões do lúpus cutâneo subagudo costumam responder bem ao uso de antimaláricos isolados ou em combinação ${ }^{18}(\mathbf{D})$.

Na falta de resposta em três meses, ou antes, quando a lesão for muito extensa, ou quando houver progressiva piora, podese associar prednisona em dose baixa a moderada por curto período de tempo.

Nos casos em que persistirem lesões cutâneas ativas, refratárias ao esquema terapêutico anterior, a escolha do tratamento deve ser baseada nas características da paciente, como a existência ou não de contra-indicações ao uso de determinada medicação ou tipo de lesão cutânea.

A talidomida, na dose de 100-200 mg/dia com redução progressiva, mostrou-se eficaz em cerca de $75 \%$ dos casos, mas deve ser indicada somente para pacientes do sexo masculino ou para mulheres sem qualquer risco de gravidez, na pósmenopausa ou com anticoncepção definitiva ${ }^{19}(\mathbf{B})$. Nos demais pacientes, pode-se associar metotrexate na dose de $10-20 \mathrm{mg} /$ semana ${ }^{14}(\mathbf{A})$, azatioprina - I a $2 \mathrm{mg} / \mathrm{kg} / \mathrm{dia}^{20}(\mathbf{C})$, clofazimine 100 mg/dia ${ }^{21}$ (D), ou dapsona - 100 mg/dia, indicado principalmente para casos de lúpus sistêmico bolhoso ${ }^{22}(\mathbf{D})$.

\section{Comprometimento articular}

A artrite habitualmente é intermitente e não erosiva, entretanto, cerca de 10\% dos casos podem evoluir com poliartrite ou oligoartrite crônica. As artrites agudas, quando não acompanhadas de comprometimento sistêmico, podem ser tratadas com antiinflamatórios não hormonais, desde que não sejam contra-indicados $^{23}(\mathbf{D})$. Caso não haja melhora, pode-se substituir ou associar prednisona em dose baixa.

Nas artrites com evolução crônica ou com recidivas freqüentes, está indicado o uso de antimalárico, hidroxicloroquina ${ }^{24}(\mathbf{A})$ ou difosfato de cloroquina para controle da dor articular. Nos casos não responsivos ou em que os antimaláricos sejam contra-indicados, pode-se associar 10-20 mg/semana de metotrexate ${ }^{25}(\mathbf{B})$.

Na prática, em pacientes que evoluem com artrite crônica, em uma ou duas articulações, não responsivos a tratamento medicamentoso acima proposto, fazemos infiltração intra-articular com triancinolona hexocetonida, geralmente com boa resposta, mas não há nenhum estudo controlado que tenha avaliado esta conduta.

texto completo da diretriz Lúpus Eritematoso Sistêmico: Tratamento do Acometimento Cutâneo/Articular está disponível no site: www.projetodiretrizes.org.br.
Referências

I .Rus V, Hochberg MC. The epidemiology of systemic lupus erythematosus. In: Wallace DJ, Hahn BH, editors. Dubois lupus erythematosus. 6th ed. Philadelphia: Lippincott Williams \& Wilkins; 2002. p.66-83

2.Tan EM, Cohen AS, Fries JF, Masi AT, McShane DJ, Rothfield NF, et al. The 1982 revised criteria for the classification of systemic lupus erythematosus. Arthritis Rheum. 1982;25: | 27|-7

3. Hochberg MC. Updating the American College of Rheumatology revised criteria for the classification of systemic lupus erythematosus. Arthritis Rheum. 1997;40: 1725

4.Dellavance A, Gabriel-Jr A, Cintra AF, Ximenes AC, Nuccitelli B, von Mühlen CA. I Consenso Nacional para Padronização dos Laudos de FAN HEp-2. J Bras Patol Med Lab. 2002;38:207-16

5.Wallace DJ. Principles of therapy and local measures. In: Wallace DJ, Hahn BH, editors. Dubois lupus erythematosus. 6th ed. Philadelphia: Lippincott Williams \& Wilkins; 2002. p. | | 3 |-47.

6. Meinao IM. Sato El, Andrade LE, Ferraz MB. Atra E. Controlled trial with chloroquine diphosphate in systemic lupus erythematosus. Lupus. 1996;5:237-4 I 7.The Canadian Hydroxychloroquine Study Group. A randomized study of the effect of withdrawing hydroxychloroquine sulfate in systemic lupus erythematosus. N Engl J Med. 1991;324: | 50-4.

8.Petri M, Lakatta C, Magder L, Goldman D. Effect of prednisone and hydroxychloroquine on coronary artery disease risk factors in systemic lupus erythematosus: a longitudinal data analysis. Am J Med. 1994;96:254-9.

9.Borba EF, Bonfa E. Long term beneficial effect of chloroquine diphosphate on lipoprotein profile in lupus patients with and without steroid therapy. J Rheumatol. 2001; 28:780-5.

I0.Wallace DJ, Linker-Israeli M, Metzger AL, Stecher VJ. The relevance of antimalarial therapy with regard to thrombosis, hypercholesterolemia and cytokines in SLE. Lupus. 1993;2(Suppl I):SI3-5.

| | . Kirou KA, Boumpas DT. Systemic glucocorticoid therapy in systemic lupus erythematosus. In: Wallace DJ, Hahn BH, editors. Dubois lupus erythematosus. $6^{\text {th }}$ ed. Philadelphia: Lippincott Williams \& Wilkins; 2002. p. I 173-94.

12.Boumpas DT, Chrousos GP, Wilder RL, Cupps TR, Balow JE. Glucocorticoid therapy for immune-mediated diseases: basic and clinical correlates. Ann Intern Med. 1993; | 19: | |98-208.

I 3. Maccune WJ, Riskalla M. Immunossuppressive drug therapy. In: Wallace DJ, Hahn BH, editors. Dubois lupus erythematosus. 6th ed. Philadelphia: Lippincott Williams \& Wilkins; 2002. p. I |95-217.

14.Carneiro JR, Sato El. Double blind, randomized, placebo controlled clinical trial of methotrexate in systemic lupus erythematosus. J Rheumatol. 1999; 26: $1275-9$.

15.Walchner M, Messer G, Kind P. Phototesting and photoprotection in LE. Lupus. 1997;6: 167-74.

16.Vila LM, Mayor AM, Valentin AH, Rodriguez SI, Reyes ML, Acosta E, et al. Association of sunlight exposure and photoprotection measures with clinical outcome in systemic lupus erythematosus. P R Health Sci J 1999; I 8:89-94.

17. Maggio KL, Singer MT, James WD. Clinical pearl: discoid lupus erythematosus - treatment with occlusive compression. J Am Acad Dermatol 1996:35:627-8

18. Costner MI, Sontheimer RD, Provost TT. Lupus erythematosus. In: Sontheimer RD, Provost TT, editors. Cutaneous manifestations of rheumatic diseases. Philadelphia: Lippincott Williams \& Wilkins; 2003. p. I5-64.

19.Atra E, Sato El. Treatment of the cutaneous lesions of systemic lupus erythematosus with thalidomide. Clin Exp Rheumatol. 1993; I 1:487-93.

20.Callen JP, Spencer LV, Burruss JB, Holtman J. Azathioprine: an effective, corticosteroid-sparing therapy for patients with recalcitrant cutaneous lupus erythematosus or with recalcitrant cutaneous leukocytoclastic vasculitis. Arch Dermatol. 1991; | 27:5 | 5-22.

21. Mackey JP, Barnes J. Clofazimine in the treatment of discoid lupus erythematosus. Br J Dermatol. 1974;91:93-6

22. Hall RP, Lawley TJ, Smith HR, Katz SI. Bullous eruption of systemic lupus erythematosus: dramatic response to dapsone therapy. Ann Intern Med. 1982;97: | 65-70.

23. Yousefi K, Weisman MH. Salicylate and nonsteroidal therapy. In: Wallace DJ, Hahn $\mathrm{BH}$, editors. Dubois lupus erythematosus. 6th Philadelphia: Lippincott Williams \& Wilkins; 2002. p. | | 4 |-7.

24.Williams HJ, Egger MI, Singer JZ, Willkens RF, Kalunian KC, Clegg DO, et al. Comparison of hydroxychloroquine and placebo in the treatment of the arthropathy of mild systemic lupus erythematosus. J Rheumatol. 1994;21 : | 457-62.

25. Rahman P, Humphrey-Murto S, Gladman DD, Urowitz MB. Efficacy and tolerability of methotrexate in antimalarial resistant lupus arthritis. J Rheumatol. 1998;25:243-6. 